\title{
Effects of RNA interference-mediated NRP-1 silencing on the proliferation and apoptosis of breast cancer cells
}

\author{
ZHENGXIANG HAN $^{1}$, GUAN JIANG ${ }^{2,3}$, YINGYING ZHANG ${ }^{1}$, JIE XU ${ }^{1}$, \\ CHONG CHEN $^{1}$, LANSHENG ZHANG $^{4}$, ZHENYUAN XU $^{1}$ and XIUPING DU ${ }^{1}$ \\ ${ }^{1}$ Department of Oncology, The Affiliated Hospital of Xuzhou Medical College; \\ ${ }^{2}$ Jiangsu Key Laboratory of Biological Cancer Therapy, Xuzhou Medical College, Xuzhou, Jiangsu 221002; \\ ${ }^{3}$ Department of Dermatology, Center for Disease Control and Prevention of Xuzhou City, Xuzhou, Jiangsu 221006; \\ ${ }^{4}$ Department of Radiology, The Second Affiliated Hospital of Xuzhou Medical College, Xuzhou, Jiangsu 221002, P.R. China
}

Received January 11, 2014; Accepted February 13, 2015

DOI: $10.3892 / \mathrm{mmr} .2015 .3405$

\begin{abstract}
Lentiviral expression vectors carrying human NRP-1 short hairpin RNA (shRNA) were constructed and selected to present highly efficient NRP-1/shRNA interference sequences, in order to investigate the effects of RNA interference (RNAi)-mediated NRP-1 silencing on the biological activities of breast cancer cells. Three pairs of human NRP-1 targeted specific interference sequences and one pair of non-specific control sequences were designed, synthesized and subcloned into pLB lentiviral vectors, which were further identified by polymerase chain reaction (PCR) and sequencing. Recombinant and lentiviral packaging plasmids were co-transfected into 293FT cell lines in order to produce lentiviral particles and to infect breast cancer cells with high NRP-1 expression. Flow cytometry was used to sort green fluorescent protein-positive cells. Fluorescence quantitative-reverse transcription-PCR and western blot analysis were employed to identify the interference silencing sequence with the most efficient silencing profile. A cell counting kit- 8 assay and an Annexin V-propidium iodide method in combination with flow cytometry were used to examine the effects of RNA interference-mediated NRP-1 gene silencing on cell proliferation, apoptosis and sensitivity to chemotherapy. The recombinant lentiviral plasmid pLB-NRP-1/shRNA was constructed successfully, as confirmed by PCR and sequencing. After the infection of recombinant lentiviral plasmids, the expression profiles of NRP-1 mRNA, and proteins of MCF-7 and SK-BR-3 cell-specific interference group (pLB-NRP-1/shRNA3) were significantly lower than that of the control group $(\mathrm{P}<0.05)$. Compared with the control group, the MCF-7 and SK-BR-3 cell-specific interference group (pLB-NRP-1/shRNA3)
\end{abstract}

Correspondence to: Dr Zhengxiang Han, Department of Oncology, The Affiliated Hospital of Xuzhou Medical College, 99 West Huai-Hai Road, Xuzhou, Jiangsu 221002, P.R. China E-mail: 5721268@163.com

Key words: short hairpin RNA, lentivirus, NRP-1, breast cancer cells, proliferation, apoptosis showed lower optical density values and higher apoptotic rates at 48,72 and $96 \mathrm{~h}$; these differences were statistically significant $(\mathrm{P}<0.05)$. EPI administration resulted in increased apoptosis in the MCF-7 and SK-BR-3 cell-specific interference groups compared with the control group $(\mathrm{P}<0.05)$. Lentiviral vectors encoding the human NRP-1 gene were constructed successfully and highly efficient NRP-1/shRNA interference sequences were selected. Furthermore, RNA interference (RNAi)-mediated NRP-1 silencing may induce proliferation suppression, apoptosis promotion, as well as enhanced sensitivity to chemotherapeutic agents.

\section{Introduction}

Breast cancer is a major threat to the health of females and accounts for 7-10\% of all incidences of systematic malignancies in China; which is continuously increasing (1). There are a number of various available interventions and $\sim 80 \%$ of patients survive for $>5$ years (2), however, breast cancer remains one of the leading causes of mortality in females. Vascular endothelial growth factor (VEGF) has been recognized to be one of the most potent pro-angiogenic factors. High expression of VEGF has been observed in breast cancer, and was shown to be correlated with prognosis (3).

NRP-1 was first identified from the axons of nerve fibers in 1995 (4), and is a $130-135 \mathrm{kDa}$ transmembrane glycoprotein, which consists of an extracellular region of 860 amino acids, a transmembrane region of 23 amino acids and an intracellular region of 40 amino acids. The extracellular region consists of five domains: a1/a2, bl/b2 and ca1/a2, which are designated to be CUB domains; and a1/a2 and b1/b2 domains, which bind to the SEMA 3 family and VEGF family, respectively (5).

NRP-1 was described to be a receptor corresponding to SEMA that may regulate the development of the nervous system (6). Recently, NRP-1 has also been demonstrated to be involved in the regulation of vascular endothelial cell migration and angiogenesis (7). Soker et al (8) observed that co-expression of VEGFR2 and NRP-1 in porcine aortic endothelial cells could increase the binding affinity of VEGF165 to VEGFR2, by at least 4-fold. This observation suggested that NRP-1 could promote the binding of VEGF165 to VEGFR2, and promote VEGFR2 
signal transduction, thereafter increasing the chemotaxis of vascular endothelium. Thus, NRP-1 has also been proposed to be a critical factor for the regulation of angiogenesis.

NRP-1 is expressed in a number of types of malignancies, such as pancreatic, lung, prostate, ovarian and gastrointestinal cancer, and it was observed to promote angiogenesis, tumor growth, invasion and metastasis (9-11). Wang et al (12) observed overexpression of NRP-1 in breast cancer tissues compared with healthy breast tissue. However, the effects of NRP-1 on the biological activities of breast cancer cells have never been reported. In this study, the effects of stable suppression of NRP-1 expression on the biological activities of MCF-7 and SK-BR-3 breast cancer cells were examined.

\section{Materials and methods}

Cell lines and reagents. MCF-7 cells were supplied by Nanjing KGI Biotechnology Company (Nanjing, China). SK-BR-3 cells were purchased from Shanghai Cell Bank of Chinese Academy of Sciences (Shanghai, China). pLB lentiviral transfer vector, pCMV $\Delta 8.91$ packaging plasmid and pMD.G envelope protein plasmids were provided by Professor Xu Kailin (Xuzhou Medical College, Xuzhou, China). Interference sequence NRP-1/short hairpin (sh)RNA was synthesized by Invitrogen Life Technologies (Carlsbad, CA, USA). Restriction endonuclease $H p a \mathrm{I}$, restriction endonuclease XhoI, and T4DNA ligase were purchased from NEB Co., USA. A fluorescence quantitative polymerase chain reaction kit was purchased from Roche Diagnostics (Indianapolis, IN, USA). Rabbit Antihuman NRP-1 polyclonal antibody was purchased from Abcam (Cambridge, UK). Epirubicin is supplied by Pfizer (USA); cell counting kit-8 kit was purchased from Dojindo Laboratories (Kumamoto, Japan). An Annexin V/propidium iodide apoptosis kit was purchased from Beijing Biosea Biotechnology Co., Ltd (Beijing, China).

Culture of cell lines. MCF-7, SK-BR-3 and 293FT human breast cancer cell lines (Invitrogen Life Technologies, Carlsbad, CA, USA), were cultured in Dulbecco's modified Eagle's medium (DMEM; Invitrogen Life Technologies) containing 10\% fetal bovine serum (Invitrogen Life Technologies) under $37^{\circ} \mathrm{C} / 5 \%$ $\mathrm{CO}_{2}$ /saturated humidity conditions in an incubator until grown to $80-90 \%$ confluency and passaged according to the principle of passage (1:2 or 1:3). When stable growth was achieved, cells in their logarithmic growth phase were selected for follow-up studies.

Design of short hairpin (sh)RNA sequences. According to the full sequence of NRP-1 mRNA retrieved from GeneBank (http://www.ncbi.nlm.nih.gov), three pairs of shRNA sequences were selected based on the principle for shRNA design. Another non-specific control sequence was also designed. All sequences designed were submitted to Invitrogen Life Technologies and the insertion sequences were described as follows: 5'-AACCCCAACAGCCTTGAATGCACTTATATTCAAGAGATATAAGTGCATTCAAGGCTGTTGGGTTTTTTC-3';5'-A ACCATTGGGCGTTACTGTGGACAGAAATTCAAGAGA TTTCTGTCCACAGTAACGCCCAATGTTTTTTC-3'; 5'-AAC CAGATCACAGCTTCTTCCCAGTATATTCAAGAGATAT ACTGGGAAGAAGCTGTGATCTGTTTTTTC-3'; and
5'-AACGTTCTCCGAACGTGTCACGTTTCAAGAGAACGTG ACACGTTCGGAGAATTTTTTC-3'. These sequences were designated shRNA1, 2, 3 and 4, respectively.

Construction and identification of lentiviral vectors carrying an RNAi sequence targeting human NRP-1 gene. The annealing products of sense and antisense strands of $\mathrm{pLB}$ and NRP-1/shRNA were treated with restriction endonucleases $H p a \mathrm{I}$ and $\mathrm{XhoI}$. Subsequent to purification, ligation, transformation and inoculation (13), they were cultured at $37^{\circ} \mathrm{C}$ overnight. Isolated colonies were selected and inoculated with ampicillin-resistant LB medium, followed by shaking at $220 \mathrm{rpm} / \mathrm{min}$ at $37^{\circ} \mathrm{C}$ overnight to extract the recombinant plasmids. After initial PCR identification, they were submitted to Invitrogen Life Technologies for sequencing identification.

Identification of breast cancer cell lines with high NRP-1 expression. Western blot analysis was used to identify the breast cancer cell lines with high NRP-1 expression, using $\beta$-actin as an internal reference. MCF-7 and SK-BR-3 breast cancer cell lines in their logarithmic growth phase were treated to extract total proteins. Equal quantities of protein were analyzed by polyacrylamide gel electrophoresis, transferred to nitrocellulose membranes under the condition of $300 \mathrm{~mA}$ current for $2 \mathrm{~h}$, and blocked with gelatin blocking solution for $1 \mathrm{~h}$. Rabbit anti-human NRP-1 polyclonal antibody (Abcam, St. Louis, MO, USA; 1:1,000 dilution) and mouse anti-human $\beta$-actin polyclonal antibody (Beijing Zhongshan Golden Bridge Biotechnology Co.,. Ltd., Beijing, China; 1:500 dilution) were added, respectively, and incubated at $4^{\circ} \mathrm{C}$ overnight. The membrane was washed with washing buffer three times for $5 \mathrm{~min}$, prior to the addition of goat anti-rabbit and horse anti-mouse antibody (Beijing Zhongshan Golden Bridge Biotechnology Co.,. Ltd., Beijing, China; 1:1,000 dilution), and the membrane was then was incubated at room temperature for $1 \mathrm{~h}$. After washing with washing buffer a further three times for $5 \mathrm{~min}$, the membrane was impregnated in BCIP/NBT (Promega, Madison, WI, USA) in order to develop color.

Packing and concentrating lentivirus. 293FT cells $\left(4 \times 10^{6}\right)$ in the logarithmic growth phase were inoculated to $100 \mathrm{~mm}$ culture plates treated with polylysine and incubated at $37^{\circ} \mathrm{C} / 5 \%$ $\mathrm{CO}_{2}$ for $1 \mathrm{~h}$. The plasmids (12 $\mu \mathrm{g}$ pLB-NRP-1/shRNA), pCMV $\Delta 8.918 \mu \mathrm{g}$ and pMD.G $4 \mu \mathrm{g}$, were diluted in $1.5 \mathrm{ml}$ serum-deprived Opti-MEM medium. Following incubation for 5 min, diluted plasmids were mixed with Lipofectamine 2000 (Invitrogen Life Technologies) prior to cultivation for $25 \mathrm{~min}$ at room temperature. Post-incubation plasmids and lipofectamine mixtures were transferred into cell cultures and mixed gently. After cultivation at $37^{\circ} \mathrm{C} / 5 \% \mathrm{CO}_{2}$ for $6 \mathrm{~h}$. Culture medium was replaced with DMEM containing $2 \% \mathrm{FBS}$ and cultivated for another $18 \mathrm{~h}$. Viral supernatants were collected at 48 and $72 \mathrm{~h}$, passed through a $0.45 \mu \mathrm{m}$ filter and centrifuged at 70,000 x g at $4^{\circ} \mathrm{C}$ for $2 \mathrm{~h}$. The supernatant was discarded and the virus particles were re-suspended in $0.5 \mathrm{ml}$ Opti-MEM medium (Invitrogen Life Technologies) and stored at $-80^{\circ} \mathrm{C}$ for further use.

Infection of lentivirus into MCF-7 and SK-BR-3 human breast cancer cells. MCF-7 and SK-BR-3 human breast cancer cells 
in their logarithmic growth phase were inoculated in 6-well plates, respectively, and cultured at $37^{\circ} \mathrm{C} / 5 \% \mathrm{CO}_{2}$ under normal conditions. Concentrated lentivirus (three replicate wells each) were added according to 3 multiplicities of infection (MOI) and diluted with polybrene to a final concentration of $8 \mu \mathrm{g} / \mathrm{l}$. Green fluorescent protein (GFP) expression profiles were examined under a fluorescence microscope (Olympus, Japan) at 24 and $48 \mathrm{~h}$ after virus infection. After enrichment culture of post-infection positive cells, flow cytometry was used to sort GFP-positive cells in the Hematology Laboratory of Soochow University (Suzhou, China), which were enriched for further experiments.

NRP-1 expression profile analyzed by fluorescent qRT-PCR and western blot analysis. MCF-7 and SK-BR-3 breast cancer cell lines, were divided into subgroups of blank controls, negative controls and specific interference groups 1,2 and 3.

Total RNA was extracted by TRIzol (Invitrogen) and treated with DNase I (New England Biolabs), and then cDNA was synthesized using GoScript Reverse Transcriptase (Promega, Madison, WI, USA) with oligodT18 primer. For quantitative PCR, mRNA expression was measured using SYBR Green I Master (Roche). The relative quantification of mRNA levels was performed using the comparative $\mathrm{Ct}$ method ( $\Delta / \Delta \mathrm{Ct}$ method) with $\beta$-actin as the reference gene. The following primers were used: NRP-1, sense 5'-GGAAGCTCTGGGCATGGAAT-3' and antisense 5'-AGGAATCCTCTCCGGGAGTC-3'; $\beta$-actin, sense 5'-CATGTACGTTGCTATCCAGGC-3' and antisense 5'-CTCCTTAATGTCACGCACGAT-3'. The PCR reaction conditions were specified as denaturation at $95^{\circ} \mathrm{C}$ for $5 \mathrm{~min}$, $95^{\circ} \mathrm{C}$ for $20 \mathrm{sec}$ and $60^{\circ} \mathrm{C}$ for $15 \mathrm{sec}$, for a total of 40 cycles. After extraction of total proteins, equal quantities of proteins were transferred to undergo SDS-PAGE analysis.

Cell proliferation by CCK-8 assay. MCF-7 and SK-BR-3 breast cancer cell lines were divided into subgroups of blank controls, negative controls and interference groups with the highest silencing efficiency.

Cells $\left(2.5 \times 10^{4} / \mathrm{ml}\right)$ from all six groups were inoculated into 96-well plates with three replicate wells and blank control wells (with medium only) for each group. Phosphate-buffered saline (PBS) was added along an arc of the plate periphery. The medium was removed after cultivation for $24,48,72$ and 96 h, respectively. Serum-deprived DMEM medium was added to each well, followed by the addition of $10 \mu \mathrm{l} \mathrm{CCK-8}$ reagent. After cultivation for another $2 \mathrm{~h}$, the optical density values at $450 \mathrm{~nm}$ wavelength were determined on a Universal Microplate Spectrophotometer (Thermo Multiskan MK3; Thermo Fisher Scientific, Waltham, MA, USA). Correct zero value from blank well to plot cell growth curve of each group.

Apoptosis examination by Annexin V-fluorescein isothiocyanate (FITC)/propidium iodide (PI) methods in combination with flow cytometry. MCF-7 and SK-BR-3 breast cancer cell lines were divided into subgroups of blank controls, negative controls and interference groups with the highest silencing efficiency.

Cells $\left(2.5 \times 10^{4} / \mathrm{ml}\right)$ of all six groups in their logarithmic growth phase were cultured for $48 \mathrm{~h}$ and apoptosis was examined using flow cytometry (BD Bioscience, San Jose, CA,
USA), according to the instructions of the Annexin V-FITC/PI kit.

\section{EPI-induced apoptosis}

Effects of different concentrations of EPI on the proliferation of MCF-7 and SK-BR-3 assayed by CCK-8. MCF-7 and SK-BR-3 breast cancer cells $\left(1.0 \times 10^{5} / \mathrm{ml}\right)$, were inoculated into 96-well plates with three replicate wells and blank control wells (with medium only) for each group. PBS was added along an arc of the plate periphery. The medium was removed after cultivation for $24 \mathrm{~h}$. Serum-deprived DMEM medium containing $0.25,0.5,1.0,2.0,4.0$ and $10.0 \mu \mathrm{g} / \mathrm{ml}$ EPI was added to each test group. Three parallel replicates were established for each group. A blank control group (free of cells) and a negative control group (containing cells and no test substance) were also analyzed. Following cultivation for $24 \mathrm{~h}$, $10 \mu \mathrm{l}$ CCK-8 reagent was added to each well and cultivated for another $2 \mathrm{~h}$. The OD values were determined at $450 \mathrm{~nm}$ wavelength on a Universal Microplate Spectrophotometer. Correct the zero value according to the blank well and depict cell growth curve of each group.

EPI induced apoptosis. In this study, MCF-7 and SK-BR-3 control groups and cell groups with the most efficient silencing NRP-1 were established. Cells in the logarithmic growth phase $\left(2 \times 10^{5}\right.$ cells/well) were inoculated to 6 -well plates separately and cultured for 48 h. MCF-7 cells were inoculated with serum-deprived medium containing $2.0 \mu \mathrm{g} / \mathrm{ml}$ EPI and SK-BR-3 cells were inoculated with serum-deprived medium containing $1.5 \mu \mathrm{g} / \mathrm{ml}$ EPI. Three replicates were used for each group. After cultivation for $24 \mathrm{~h}$, apoptosis was examined according to the manufacturer's instructions of the Annexin V-FITC/PI kit in combination with flow cytometry.

Statistical analysis. Statistical analyses were conducted using SPSS 16.0 (SPSS, Inc., Chicago, IL, USA). Quantitative data are represented as the mean \pm standard deviation. Pair-wise inter-group comparison was analyzed using a t-test. Multiple group comparisons were analyzed using a one-way analysis of variance test. $\alpha=0.05$ and $\mathrm{P}<0.05$ were considered to indicate a statistically significant difference.

\section{Results}

Virus infection and flow cytometry of MCF-7 and SK-BR-3 cells. MCF-7 and SK-BR-3 cells were infected for $48 \mathrm{~h}$ and only low GFP expression was observed under fluorescence microscopy. GFP positive cells were sorted and re-examination suggested a positive rate of $96 \%$ after sorting (Figs. 1 and 2).

Significant expression suppression of NRP-1 mRNA and protein following virus infection. Blank control, negative control and specific interference groups (NRP-1/shRNA1, NRP-1/shRNA2, and NRP-1/shRNA3) were established in this study. With $\beta$-actin as the internal reference, the relative expression profiles of target gene was estimated based on $2^{-\Delta \Delta \mathrm{Ct}}$. Compared with the control group, the expression profiles of NRP-1 mRNA and protein were decreased 


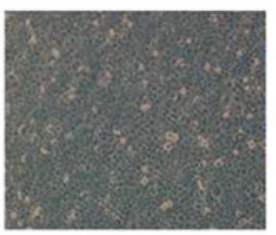

A

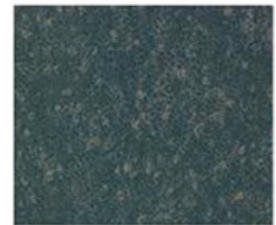

E

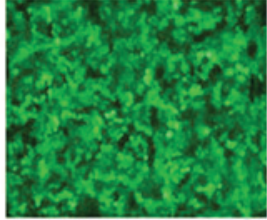

B

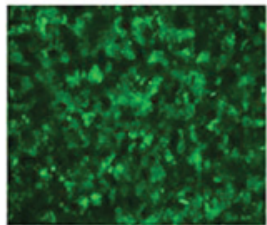

F

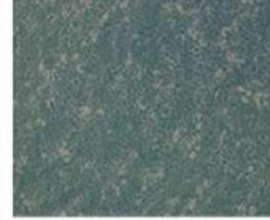

C

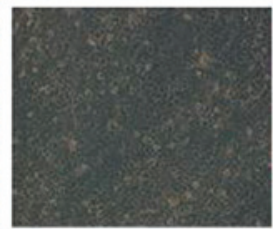

G

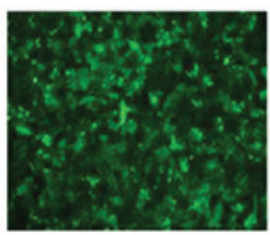

$\mathrm{D}$

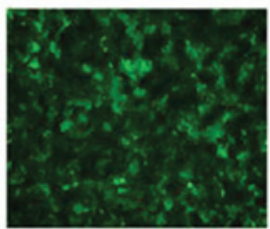

$\mathrm{H}$

Figure 1. Expression of GFP in MCF-7 cells after virus infection and flow sorting under fluorescence microscopy (x100). (A) pLB-NRP-1/shRNA controls in optical microscopy; (B) pLB-NRP-1/shRNA controls in fluorescent microscopy; (C) pLB-NRP-1/shRNA1 in optical microscopy; (D) pLB-NRP-1/shRNA1 in fluorescent microscopy; (E) pLB-NRP-1/shRNA2 in optical microscopy; (F) pLB-NRP-1/shRNA2 in fluorescent microscopy; (G) pLB-NRP-1/shRNA3 in optical microscopy; (H) pLB-NRP-1/shRNA3 in fluorescent microscopy. GFP, green fluorescent protein; shRNA, short hairpin RNA.

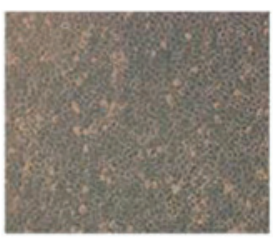

A

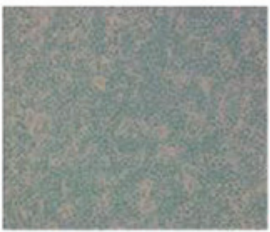

E

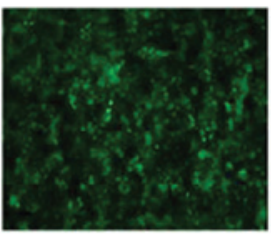

B

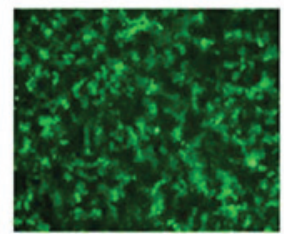

F

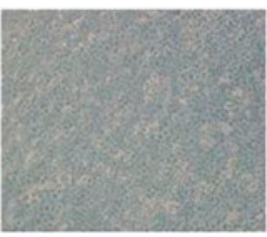

C

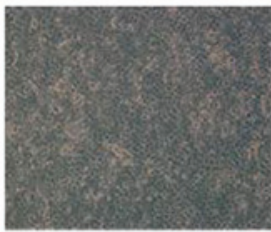

G

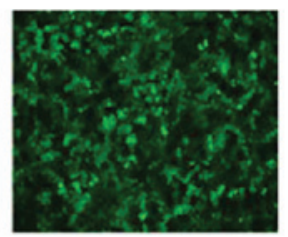

D

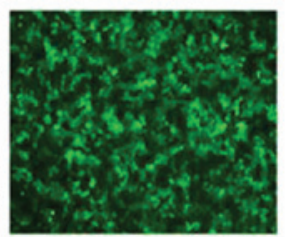

H

Figure 2. Expression of GFP in SK-BR-3 cells after virus infection and flow sorting under fluorescence microscopy (x100). (A) pLB-NRP-1/shRNA controls in optical microscopy; (B) pLB-NRP-1/shRNA controls in fluorescent microscopy; (C) pLB-NRP-1/shRNA1 in optical microscopy; (D) pLB-NRP-1/shRNA1 in fluorescent microscopy; (E) pLB-NRP-1/shRNA2 in optical microscopy; (F) pLB-NRP-1/shRNA2 in fluorescent microscopy; (G) pLB-NRP-1/shRNA3 in optical microscopy; (H) pLB-NRP-1/shRNA3 in fluorescent microscopy. GFP, green fluorescent protein; shRNA, short hairpin RNA.
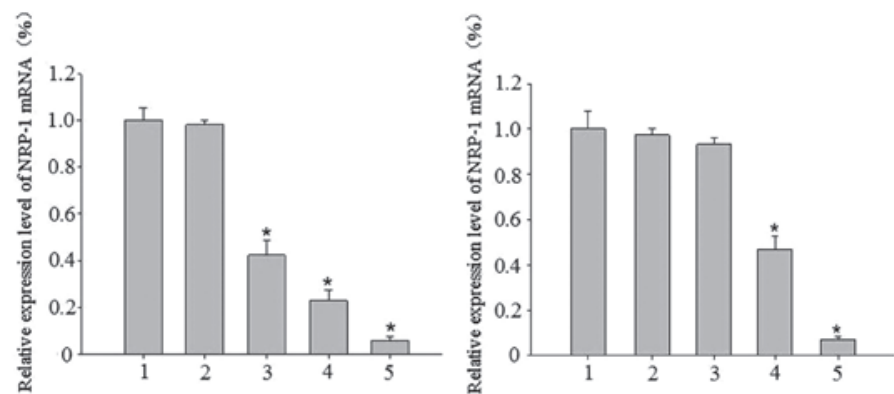

1. Blank control group: 2 . Negative control group 3 , shRNA1;4, shRNA2;5, shRNA3

MCF-7

SK-BR-3

Figure 3. Relative expression level of NRP-1 mRNA after lentiviral infection. ${ }^{*} \mathrm{P}<0.05$, compared with the control group.

significantly in the MCF-7 cell-specific interference groups (pLB-NRP-1/shRNA1, 2 and 3), with mRNA expression decreased by $57.73,77.20$ and $94.17 \%$ and protein expression decreased by $15.85,26.25$ and $49.29 \%$, respectively. For the
SK-BR-3 cell specific interference group (pLB-NRP-1/shRNA1, 2 and 3), the expression profiles of NRP-1 mRNA and protein also were also significantly decreased, with mRNA expression decreased by 53.20 and $92.94 \%$, protein expression decreased 


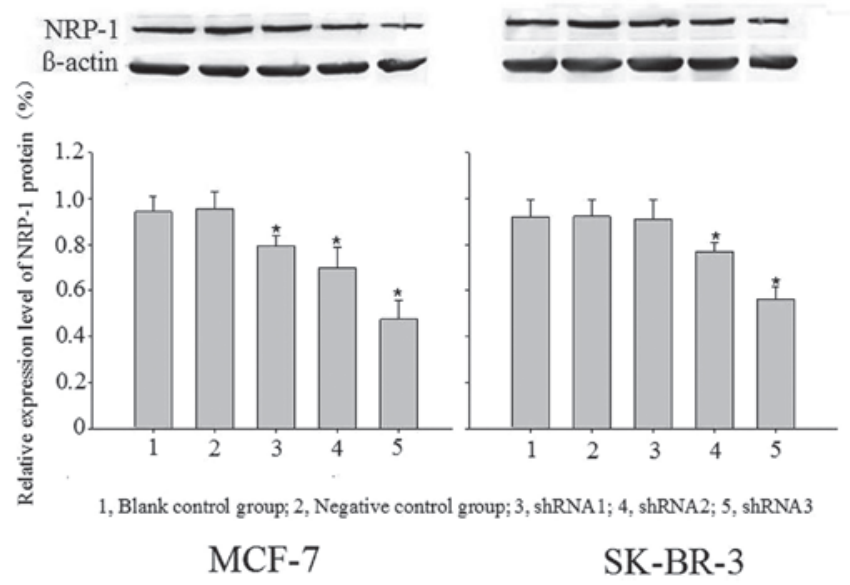

Figure 4. Relative expression level of NRP-1 protein after lentiviral infection. " $\mathrm{P}<0.05$, compared with the control group. shRNA, short hairpin RNA.

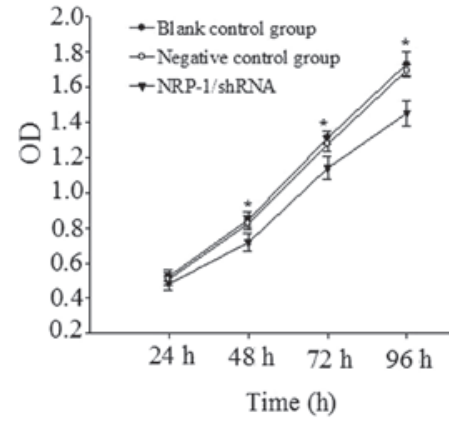

MCF-7

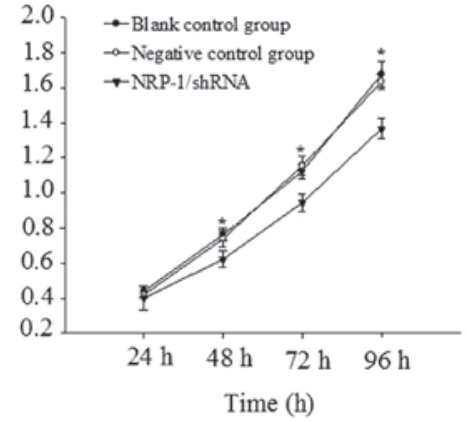

SK-BR-3

Figure 5. Effect of NRP-1 intervention on the proliferation of breast cancer cells. "P<0.05, compared with the control group. shRNA, short hairpin RNA.

MCF-7
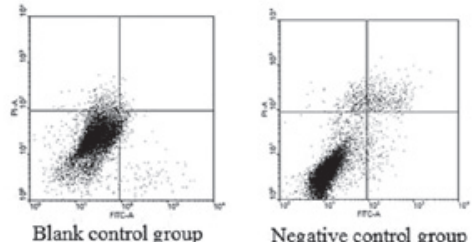

Negative control group

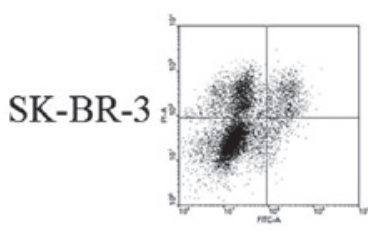

Blank control group

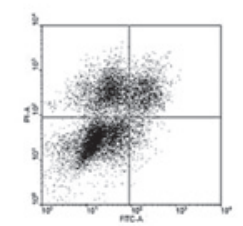

Negative control group

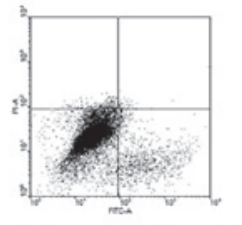

NRP-1 intervention group

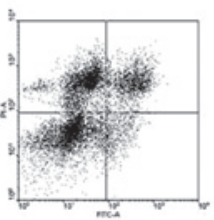

NRP-1 intervention group

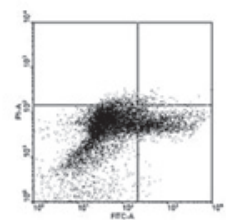

Control group after the treatment of EPI

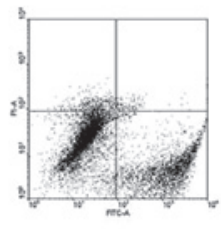

Control group after the treatment of EPI
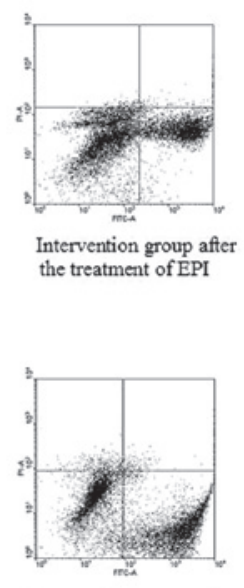

Intervention group after
the treatment of EPI

Figure 6. Detection of the apoptosis rate in MCF-7 and SK-BR-3 cells using flow cytometry. EPI, epirubicin.

by 15.98 and $38.50 \%$, respectively. These results were statistically significant compared with the control group $(\mathrm{P}<0.05)$; pLB-NRP-1/shRNA3 showed the highest silencing efficiency among these two cell lines. The pair-wise comparison between the negative control group and blank control group was not statistically significant ( $>0.05)$ (Figs. 3 and 4).
Determination of proliferation rate. According to the proliferation rates determined by the CCK- 8 method, the OD values of MCF-7 cell with NRP-1/shRNA group were determined to be $0.717 \pm 0.051,1.140 \pm 0.064$ and $1.453 \pm 0.071$ at 48,72 and $96 \mathrm{~h}$ respectively. This difference was statistically significant comparison to the control group $(\mathrm{P}<0.05)$. The OD 


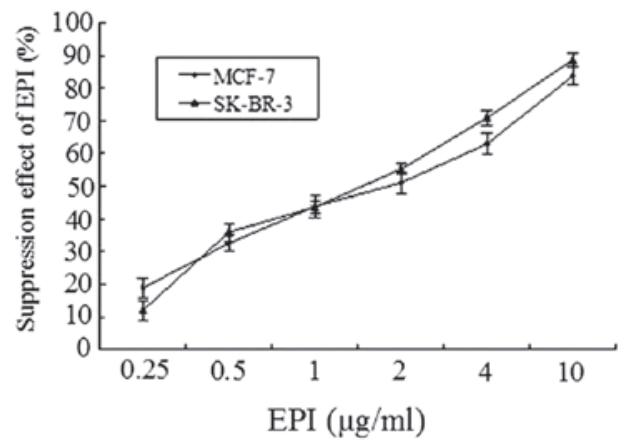

Figure 7. EPI inhibition on the growth of breast cancer cells. EPI, epirubicin.

values of SK-BR-3 cell with NRP-1/shRN were $0.622 \pm 0.046$, $0.944 \pm 0.051$ and $1.365 \pm 0.058$ at $48 \mathrm{~h}, 72 \mathrm{~h}$, and $92 \mathrm{~h}$ respectively. This difference was statistically significant compared with the control group $(\mathrm{P}<0.05)$, but the inter-group difference between the negative control group and the blank control group was not statistically significant $(\mathrm{P}>0.05)$ (Fig. 5).

Determination of apoptotic rate. The apoptotic rates of MCF7 cells determined by the Annexin V-propidium iodide method in combination with flow cytometry were $2.05 \pm 0.18,2.41 \pm 0.29$ and $9.76 \pm 0.54 \%$ for the blank control group, the negative control group and the NRP-1 intervention group; while the apoptotic rates of SK-BR-3 cells were $3.04 \pm 0.17,3.22 \pm 0.26$ and $5.98 \pm 0.17 \%$ for the blank control group, the negative control group and the NRP-1 intervention group, respectively. Compared with the control group, the rate of apoptosis of cells in the NRP-1/shRNA groups was higher $(\mathrm{P}<0.05)$ and no significant differences were observed in the negative group and blank group ( $\mathrm{P}>0.05)$ (Fig. 6).

Selection of EPI concentration. CCK-8 assays demonstrated that the suppression effect of EPI tends to increase with the elevation of concentration in the range between 0 and $10.0 \mu \mathrm{g} / \mathrm{ml}$. At $2 \mu \mathrm{g} / \mathrm{ml}$, the inhibitory rates of MCF-7 and SK-BR-3 cells were $50.99 \pm 3.07$ and $55.18 \pm 1.58 \%$ respectively. The $\mathrm{IC}_{50} \mathrm{~s}$ were determined to be 2.0 and $1.5 \mu \mathrm{g} / \mathrm{ml}$ for MCF-7 and SK-BR-3 respectively. Therefore, 2.0 and $1.5 \mu \mathrm{g} / \mathrm{ml}$ were selected as the EPI concentrations for MCF-7 and SK-BR-3 assays, respectively (Fig. 7).

Determination of EPI-induced apoptosis. As shown by the assays with Annexin V-FITC/PI in combination with flow cytometry, the apoptotic rates of MCF-7 cells with NRP-1 interference and the control group were $35.32 \pm 2.24$ and $20.80 \pm 3.08 \%$ respectively after EPI treatment, while the apoptotic rates of SK-BR-3 and the control group were $49.24 \pm 3.66$ and $34.15 \pm 1.97 \%$, respectively. The apoptotic rate of the interference group was higher than the control group and the difference was statistically significant $(\mathrm{P}<0.05)$ (Fig. 6).

\section{Discussion}

Breast cancer is the most common type of malignancy in females and remains to be a common cause of cancer-related mortality, secondary only to lung cancer (14). Although there are numerous therapies available to treat metastatic breast cancer, poor responses are observed for the majority of patients with malignancies (15). Therefore, current research is focused on relevant targeted therapies (16). In patients with early node-positive and -negative breast cancer, the VEGF expression profile has been observed to be associated with the reduction of PFS (progression-free survival) and OS (overall survival). Thus VEGF has been recognized to be an effective target for anti-angiogenic therapies (17). Furthermore, the anti-VEGF monoclonal antibody, bevacizumab has been approved by the FDA to treat breast cancer (18).

However, the results of AVADO and RIBBON-1 trials demonstrated PFS other than OS could benefit from that the treatment of bevacizumab in combination with cytotoxic chemotherapy $(19,20)$. The earlier AVF2119g trial, evaluating the addition of bevacizumab to capecitabine in first to fifth-lineMBC (including patients relapsing on therapy), demonstrated a $10.7 \%$ improvement in response rate $\left(\mathrm{P}^{1 / 40.001)}\right.$ and no PFS benefit (21). Importantly, bevacizumab have sideeffects, the most important being hypertension,proteinuria, bleeding and thromboembolic events. Some concerns have also been raised that bevacizumab might increase the rate of heart failure in breast cancer patients (22). Therefore, bevacizumab has limited utility for the treatment of breast cancer due to its limited efficacy.. Therefore, bevacizumab has limited utility for the treatment of breast cancer due to its limited efficacy. The utility of anti-angiogenic therapy in the treatment of breast cancer requires further evaluation. Investigation into the mechanism underlying anti-angiogenic therapies in breast cancer may facilitate the development and application of targeted therapies and individualized treatment.

As a multifunctional non-tyrosine kinase receptor, NRP-1 is expressed in arterial endothelial cells and tumor cells. NRP-1 expression profiles have been correlated with the progress and prognosis of malignancies, while NRP-2 is predominantly expressed in veins and lymphatic endothelium (23-25). In 1998, Soker et al (8) purified NRP-1 from human breast cancer cell lines and disclosed the important roles of NRP-1 in the metastatic process of breast cancer. Ghosh et al (23) demonstrated that NRP-1 was highly expressed in metastatic breast cancer. Another study also demonstrated that the NRP-1 expression profile of breast cancer cell lines with high metastatic potential in vitro was higher than that in lines with low metastatic potential (12). High expression of NRP-1 and VEGF in breast tissues was closely associated with poor prognosis (23). This result suggested that NRP-1 may be important for the biological behavior of breast cancer and it has been proposed to be a novel target for anti-angiogenic and individual therapies. Therefore, further investigation into the association between NRP-1 and the biological behavior of breast cancer.

In this study, MCF-7 and SK-BR-3 breast cancer cell lines with high NRP-1 expression were selected. Lentiviral vectors encoding the human NRP-1 gene were successfully constructed based on lentiviral vectors carrying interference sequences. Highly efficient NRP-1/shRNA interference sequences were selected. The suppression of NRP-1 mRNA and protein mediated by NRP-1/shRNA lentivirus resulted in inhibition of the proliferation of breast cancer cells in vitro and promotion of apoptosis. The results of this study were consistent with those reported in the literature. Ochiumi et al (26) 
demonstrated the correlation between the poor prognosis of colon cancer and high NRP-1 expression, and NRP-1 interference was observed to suppress the migration of colon cancer cells and induce apoptosis. Another study also demonstrated that the post-NRP-1 interference inhibition of proliferation and induction of apoptosis observed in glioma cells may be closely associated with the decrease of Bcl-2 family expression and inactivation of cancer development related signaling pathways, such as extracellular signal-regulated kinases, c-Jun N-terminal kinases and mitogen-activated protein kinase (27). However, the mechanisms of proliferation inhibition and apoptosis induction observed in breast cancer cells remain to be elucidated.

Chemotherapy containing anthracyclines are important regimens in the treatment of breast cancer. Myelotoxicity and cardiotoxicity have been identified to be the dose-limiting toxicities (DLT) of these medications. These deleterious effects (particularly cardiotoxicity) also restricted the improvement of their clinical efficacy. Several studies demonstrated that high NRP-1 expression has been recognized to be an independent prognostic factor for breast cancer. Therefore, whether concomitant administration of anthracyclines could increase the sensitivity to chemotherapy following NRP-1 interference required further investigation. As shown by this study, the apoptotic rate was significantly higher than that of control group after concomitant interventions of NRP-1 interference and epirubicin. This result suggested that NRP-1 interference contributed to the increase of sensitivity to epirubicin treatment. However, whether the NRP-1 targeted strategy targeting may increase the sensitivity of epirubicin resistance cell lines to chemotherapies and whether the synergic effects may exist when epirubicin is administered in combination with other chemotherapeutic agents required further investigation.

In conclusion, MCF-7 and SK-BR-3 cells with high NRP-1 protein expression were identified in this study. Lentiviral expression vectors encoding the NRP-1 gene were constructed successfully using lentiviral vectors. RNA interference significantly reduced the expression of NRP-1. In addition, NRP-1 silencing suppressed the proliferation of MCF-7 and SK-BR-3 cells, promoted apoptosis and increased their sensitivity to epirubicin chemotherapy. This evidence provides a theoretical basis for NRP-1 targeted chemotherapies against breast cancer. In this study, the effects of NRP-1 over-expression on the biological behavior of breast cancer have been investigated. As the co-receptor of VEGF165, NRP-1 is highly expressed in breast cancer and the signaling pathway of VEGFR mediated by VEGF has been extensively investigated; however, the NRP-1 signaling pathway mediated by VEGF requires further elucidation.

\section{Acknowledgements}

The authors would like to thank the Hematology Laboratory of Soochow University (Suzhou, China) for their assistance in sorting GFP-positive cells.

\section{References}

1. Yi W, Peng J, Zhang Y, et al: Differential protein expressions in breast cancer between drug sensitive tissues and drug resistant tissues. Gland Surg 2: 62-68, 2013.
2. Barrett SV: Breast cancer. J R Coll Physicians Edinb 40: 335-338, 2010.

3. Perez EA and Spano JP: Current and emerging targeted therapies for metastatic breast cancer. Cancer 118: 3014-3025, 2012.

4. Satoda M, Takagi S, Ohta K, et al: Differential expression of two cell surface proteins, neuropilin and plexin, in Xenopus olfactory axon subclasses. J Neurosci 15: 942-955, 1995.

5. Jubb AM, Strickland LA, Liu SD, et al: Neuropilin-1 expression in cancer and development. J Pathol 226: 50-60, 2012.

6. Soker S, Takashima S, Miao HQ, Neufeld G and Klagsbrun M: Neuropilin-1 is expressed by endothelial and tumor cells as an isoform-specific receptor for vascular endothelial growth factor. Cell 92: 735-745, 1998.

7. Miao HQ, Lee P, Lin H, Soker S and Klgsbrun M. Neuropilin-1 expression by tumor cells promotes tumor angiogenesis and progression. FASEB J 14: 2532-2539, 2000.

8. Soker S, Takashima S, Miao HQ, et al: Neuropilin-1 is expressed by endothelial and tumor cells as an isoform-specific receptor for vascular endothelial growth factor. Cell 92: 735-745, 1998.

9. Guttmann-Raviv N, Kessler O, Shraga-Heled N, et al: The neuropilins and their role in tumorigenesis and tumor progression. Cancer Lett 231: 1-11, 2006.

10. Karjalainen K, Jaalouk DE, Bueso-Ramos CE, et al: Targeting neuropilin-1 in human leukemia and lymphoma. Blood 117: 920-927, 2011.

11. Pan Q, Chanthery Y, Liang WC, et al: Blocking neuropilin-1 function has an additive effect with anti-VEGF to inhibit tumor growth. Cancer Cell 11: 53-67, 2007.

12. Wang ZL, Tang ZC, Zhang Y, et al: Neuropilin-1 down-regulation impairs cell migration and induces the differentiation of human tongue squamous cell carcinoma cells. Head Neck Oncol 4: 54, 2012.

13. Cao J, Meng FJ, Li L, et al: Expression of NANOG gene in acute lymphoblastic leukemia cells and construction of lentiviralvector carrying NANOG specific shRNA. Zhongguo Shi Yan Xue Ye Xue Za Zhi 22: 275-279, 2014. (In Chinese)

14. Jha K, Shukla M and Pandey M: Survivin expression and targeting in breast cancer. Surg Oncol 21: 125-131, 2012.

15. Alvarez RH, Valero V and Hortobagyi GN: Emerging targeted therapies for breast cancer. J Clin Oncol 28: 3366-3379, 2010

16. Koutras AK, Fountzilas G, Makatsoris T, et al: Bevacizumab in the treatment of breast cancer. Cancer Treat Rev 36: 75-82, 2010.

17. Goldfarb SB, Traina TA and Dickler MN: Bevacizumab for advanced breast cancer. Womens Health (Lond Engl) 6: 17-25, 2010.

18. Alvarez RH, Guarneri V, Icli F, et al: Bevacizumab treatment for advanced breast cancer. Oncologist 16: 1684-1697, 2011.

19. Miles DW, Chan A, Dirix LY, et al: Phase III study of bevacizumab plus docetaxel compared with placebo plus docetaxel for the first-line treatment of human epidermal growth factor receptor 2-negative metastatic breast cancer. J Clin Oncol 28: 3239-3247, 2010.

20. Robert NJ, Diéras V, Glaspy J, et al: RIBBON-1: randomized, double-blind, placebo-controlled, phase III trial of chemotherapy with or without bevacizumab for first-line treatment of human epidermal growth factor receptor 2-negative, locally recurrent or metastatic breast cancer. J Clin Oncol 29: 1252-1260, 2011.

21. Miller KD, Chap LI, Holmes FA, et al: Randomized phase III trial of capecitabine compared with bevacizumab plus capecitabine in patients with previously treatedmetastatic breast cancer. J Clin Oncol 23: 792-799, 2005.

22. Choueiri TK, Mayer EL, Je Y, et al: Congestive heart failure risk inpatients with breast cancer treated with bevacizumab. J Clin Oncol 29: 632-638, 2011.

23. Ghosh S, Sullivan CA, Zerkowski MP, et al: High levels of vascular endothelial growth factor and its receptors (VEGFR-1, VEGFR-2, neuropilin-1) are associated with worse outcome in breast cancer. Hum Pathol 39: 1835-1843, 2008.

24. Herzog Y, Kalcheim C, Kahane N, et al: Differential expression of neuropilin-1 and neuropilin-2 in arteries and veins. Mech 109: 115-119, 2001.

25. Yuan L, Moyon D, Pardanaud L, et al: Abnormal lymphatic vessel development in neuropilin 2 mutant mice. Development 129: 4797-4806, 2002.

26. Ochiumi T, Kitadai Y, Tanaka S, et al: Neuropilin-1 is involved in regulation of apoptosis and migration of human colon cancer. Int J Oncol 29: 105-116, 2006.

27. Li X, Tang T, Lu X, et al: RNA interference targeting NRP-1 inhibits human glioma cell proliferation and enhances cell apoptosis. Mol Med Rep 4: 1261-1266, 2011. 